\title{
Gastric collision between a papillotubular adenocarcinoma and a gastrinoma in a patient with Zollinger-Ellison syndrome
}

\author{
Laurence de Leval ${ }^{\mathrm{a}}$, Noëlle Hardy ${ }^{\mathrm{a}}$, Manuel Deprez ${ }^{\mathrm{a}}$, Jean Delwaide ${ }^{\mathrm{b}}$, Jacques Belaïche ${ }^{\mathrm{b}}$, Jacques Boniver ${ }^{\mathrm{a}}$ \\ ${ }^{a}$ Department of Pathology, CHU of Liège, Sart-Tilman,4000 Liège, Belgium \\ ${ }^{b}$ Department of Gastroenterology, CHU of Liège, Sart-Tilman,4000 Liège, Belgium
}

\begin{abstract}
We report a unique case of a gastric collision tumor composed of an intramural gastrin-secreting tumor and a papillotubular adenocarcinoma of the intestinal type discovered at autopsy in a patient with Zollinger-Ellison syndrome. There was extensive metastatic dissemination of the neuroendocrine component to regional lymph nodes and to the liver. The unusual macroscopical, histological, and immunohistochemical features of this case and its specific clinical setting are discussed
\end{abstract}

Keywords : Carcinoma-carcinoid spectrum ; Gastric collision tumor ; Intramural gastrin-secreting tumor ; Papillotubular adenocarcinoma ; Zollinger-Ellison syndrome

\section{INTRODUCTION}

Admixtures of neoplastic endocrine and nonendocrine epithelial cells have been found frequently in digestive tumors, leading to the concept of a "carcinoma-carcinoid spectrum" [8]. This spectrum encompasses four main categories: (a) carcinomas with interspersed endocrine cells, (b) carcinoids with interspersed nonendocrine cells, (c) mixed tumors comprising both carcinoma and carcinoid areas, and (d) amphicrine tumors consisting of cells exhibiting both endocrine and nonendocrine differentiation. The first category is by far the most frequent one, comprising a large number of adenocarcinomas containing varying proportions of endocrine cells scattered among mucin-secreting cells and special entities such as goblet cell carcinoids of the appendix. The category of mixed tumors can be further subdivided into composite-type tumors, showing an admixture of the two histological components with frequent histological transitions, and collision-type tumors, where the two components, while intimately juxtaposed with a "side-by-side" or "one-upon-another" pattern, are not intermixed. Collision-type tumors are usually considered as double coexistent and independent tumors. Collision tumors of the gastrointestinal tract are much less common than the composite variety; several cases have been reported in the stomach $[3,10,15]$, and in all of these cases the neuroendocrine component was either a carcinoid tumor or a malignant neuroendocrine carcinoma. To our knowledge, no such case has been associated with a specific clinical syndrome related to hormone hypersecretion.

We report here what we believe is the first case of a gastric collision tumor composed of an intramural gastrinsecreting tumor and a well-differentiated papillotubular adenocarcinoma of the intestinal type in a patient with Zollinger-Ellison syndrome (ZES).

\section{MATERIALS AND METHODS}

\section{Clinical history}

The patient came to medical attention at the age of 87 years when he presented with massive digestive bleeding due to diffuse gastric, duodenal, and jejunal ulcerations, prompting a surgical antiulcer procedure (vagotomy and pyloroplasty). During this surgery a $1-\mathrm{cm}$ intramural gastric nodule and a sessile duodenal polyp $(1.5 \mathrm{~cm})$ were incidentally found and resected. The gastric lesion proved to be a benign c-Kit positive stromal tumor, and the duodenal polyp was diagnosed as a villous adenoma with severe dysplasia. Following surgery and under antiulcer medication ( $20 \mathrm{mg} /$ day omeprazole) digestive bleeding recurred, originating from upper jejunal ulcerations. ZES was then suspected. The diagnosis was confirmed definitively by marked elevation in fasting serum gastrin level (>3500 pg/ml), elevated basal acid output ( $16 \mathrm{mmol} / \mathrm{h})$ despite the vagotomy, and 
paradoxical increase in gastrin levels and acid output (to $36 \mathrm{mmol} / \mathrm{h}$ ) after intravenous secretin infusion $(3 \mathrm{U} / \mathrm{kg}$ ). At the time of diagnosis, echoendoscopy, abdominal computed tomography with intravenous contrast, and selective celiac and superior mesenteric arteriography remained negative in the search of an abdominal tumor. Somatostatin receptor scintigraphy performed with ${ }^{111}$ In-labeled octreotide identified a single foeus of increased uptake in the upper right abdomen considered to be of duodenal or paraduodenal origin. The patient had no familial history and no associated endocrine disorders suggestive of multiple endocrine neoplasia type 1 . Due to his general condition the patient was ineligible for surgery and was kept under high doses of antisecretory medications ( $40 \mathrm{mg} /$ day omeprazole), which effectively prevented ulterior bleeding. He was readmitted to our hospital 4 years after the initial diagnosis, with cachexia, abdominal pain and clinical evidence of metastatic liver disease, confirmed by abdominal computed tomography. Surprisingly, gastros-copy revealed a large antral fungating mass, biopsy specimens of which showed in situ intestinal-type papillary adenocarcinoma. The patient died in a context of hepatorenal failure shortly after his admission. An autopsy was performed.

\section{Histology}

At the time of necropsy special attention was paid to the dissection of the upper abdomen in order to find the primary gastrinoma, to characterize the extent of the neoplastic villous gastric tumor, and to define the extent and origin of the metastatic dissemination. Tissue for histological examination was fixed overnight in $10 \%$ formalin, sectioned at $0.2-\mathrm{cm}$ intervals, and embedded in paraffin. Sections $5 \mu \mathrm{m}$ thick were stained with hematoxylineosin and by immunohistochemistry. Immunoperoxidase stains were performed using a three-step indirect immunoperoxidase technique after antigen retrieval by microwaving the slides in citrate buffer for $20 \mathrm{~min}$. Primary antibodies against the following antigens were used: monoclonal antibody neuron-specific enolase (Dako), monoclonal antibody chromogranin A (Dako), and monoclonal antibody gastrin (Immunotech).

\section{RESULTS}

Examination of the stomach revealed a 5.5-cm polypoid tumor on the greater curvature of the antrum. Cut surface showed a fungating villous pedunculated lesion and thickening of the underlying gastric wall by nodular and diffusely infiltrative whitish firm tumoral tissue (Fig. 1). There was no macroscopic extension through the serosa. The remainder of the gastric mucosa appeared smooth, and no other mucosal or intramural gastric lesion was identified. Three enlarged tumoral lymph nodes, measuring 1-4 cm in their greatest diameter, were found in the vicinity of the stomach (in retropyloric, retroduodenal, and lateroaortic locations). Most of the liver parenchyma was replaced by large metastatic tumor masses. Examination of the duodenum and pancreas was performed by serial sectioning into 3-mm slices and failed to show any tumor in these organs. Macroscopical appearance of the duodenal wall was unremarkable, with no evidence of residual or recurrent mucosal tumor. The pancreas was atrophic with ductular distension and mucoid retention. The small bowel and colon were unremarkable. There was no evidence of tumor outside the abdomen.

Histopathological examination of the gastric tumor revealed two distinct microscopic appearances. The upper exophytic part consisted of a well-differentiated papillotubular adenocarcinoma of the intestinal type with no extension through the muscularis mucosae (Fig. 2a). The intramural tumor displayed microscopic features suggestive of a neuroendocrine proliferation (Fig. 2b). It was composed of a relatively uniform population of small- to medium-sized cells with an organoid, trabecular or acinar, or focally solid pattern, and frequent rosette formation. The nuclei had homogeneous chromatin with small or indistinct nucleoli, and showed slight variation in size and shape; mitoses were infrequent $(<10 / 10 \mathrm{hpf})$ and no necrosis was seen. The latter component extensively infiltrated into submucosal and subserosal lymphatic vessels and extended into the pedicle of the upper villous tumor. The two tumor components were abutting each other with no transition between them (Fig. 2c). The neoplastic proliferation in all lymph nodes and in the liver exhibited an exclusively neuroendocrine pattern. The nontumoral gastric mucosa, macroscopically unremarkable, was found to contain exclusively fundic-type glands and showed nodular hyperplasia of enterochromaffin-like endocrine cells. There was no evidence of Helicobacter pylori gastritis or intestinal metaplasia.

A strong immunoreactivity for neuroendocrine markers (chromogranin-A, neuron-specific enolase) and positivity for the Grimelius silver stain were found in the intramural gastric tumor as well in lymph nodes and liver metastasis, whereas the gastric epithelial tumor contained only scattered neuroendocrine cells (Fig. 2d). About $15 \%$ of gastric neuroendocrine neoplastic cells and $50 \%$ of lymph nodes tumor cells displayed strong cytoplasmic immunoreactivity to gastrin (Fig. 2e). Gastrin was not detected in liver metastasis. 
Fig. 1 Macroscopic view of the gastric tumor

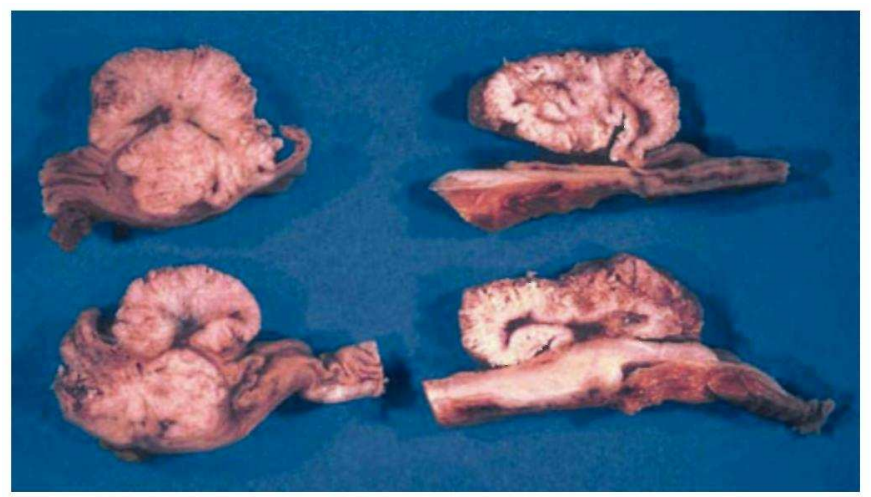

Fig. 2 a Upper villous portion of the gastric tumor showing papillo-tubular adenocarcinoma; hematoxylin and eosin, original magnification $\times 100$. b Intramural neuroendocrine portion of the gastric tumor showing rosette formation; hematoxylin and eosin, original magnification $\times 200$. c Collision area between the two portions of the tumor; hematoxylin and eosin, original magnification $\times 25$. $d$ Chromogranin immunohistochemistry of the collision area showing strong staining of the intramural neuroendocrine tumor and isolated endocrine cells in the upper adenoma; immunoperoxidase, original magnification $\times 25$. e Gastrin immunohistochemistry showing strong staining of some cells in the neuroendocrine component of the gastric tumor; immunoperoxidase, original magnification $\times 400$
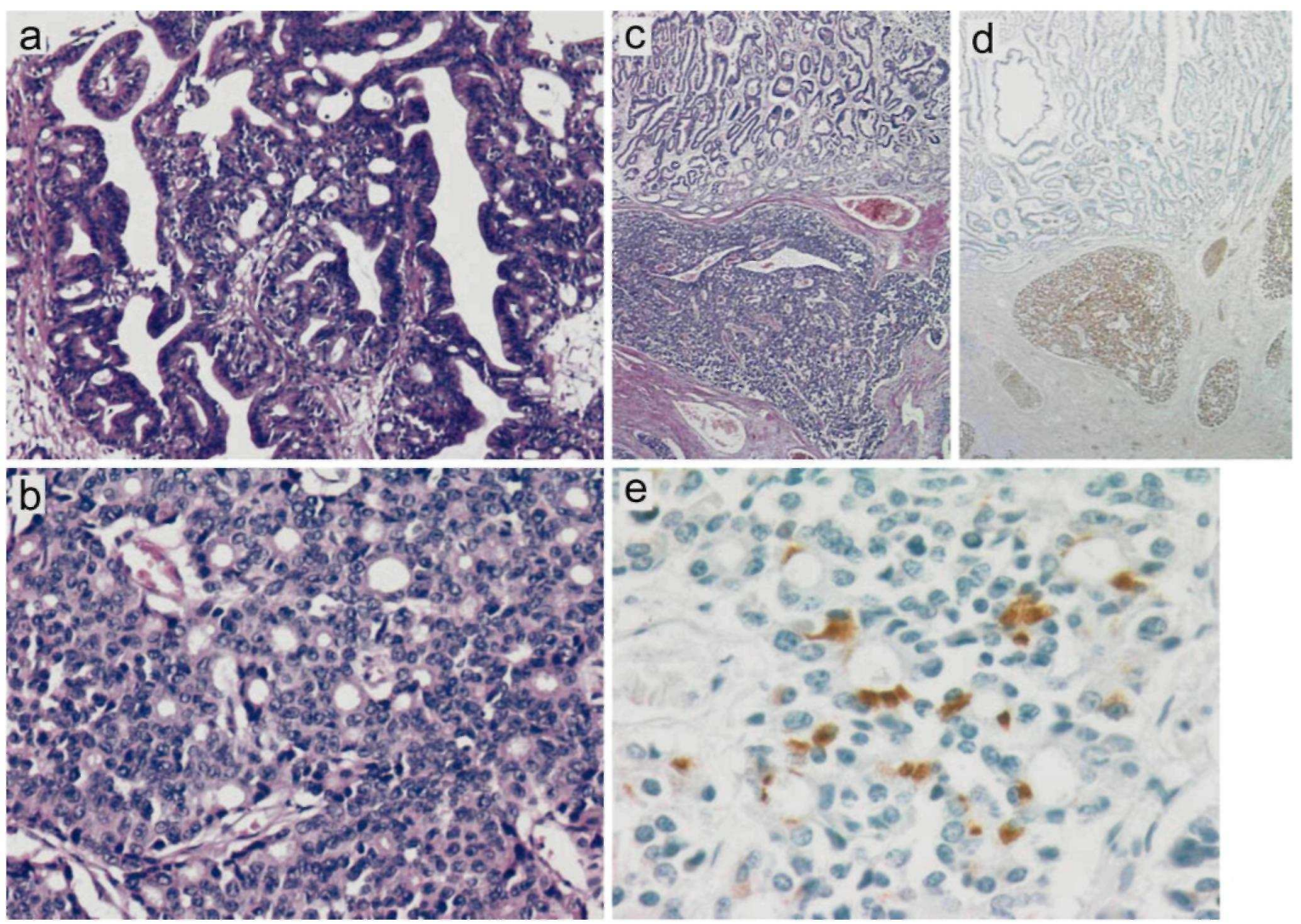

\section{DISCUSSION}

We report here the unique case of a gastric well-differentiated papillotubular adenocarcinoma of the intestinal type in collision with an intramural gastric gastrinoma. Evidence that this tumor indeed belongs to the "collisiontype" category of mixed endocrine-nonendocrine tumors is supported by the following: (a) the tumor comprised 
two components with clearly different histological and immunohistological patterns; (b) the two components were macroscopically abutting each other with no histological transition between them; the upper adenocarcinoma was strictly intramucous in contrast to the intamural extent and the extensive lymphatic permeation by the underlying neuroendocrine proliferation; and (c) lymph nodes and liver metastasis were monomorphic, displaying a neuroendocrine differentiation. To our knowledge, this case represents the first report of a collision tumor with a functional, secreting neuroendocrine component.

Another peculiar feature of this case was the finding of a gastrinoma in a gastric location. Somewhat paradoxically, the stomach, which under physiological conditions contains most of the $\mathrm{G}$ cells of the organism, appears as a very rare site of origin for gastrinomas [7, 14, 16]. A review of the literature disclosed fewer than 20 reported gastric gastrinomas presenting as a mucosal polyp or as unique or multiple intramural nodules $[9,12$, $13,14]$. The possibility of a primary paraduodenal nodal gastrinoma, which might have been considered from the scintigraphic data, was positively ruled out by the finding of a parenchymal gastrin-producing neuroendocrine tumor and the involvement of multiple locoregional lymph nodes. Interestingly, a study on the sensitivity and specificity of preoperative somatostatin receptor scintigraphy in detecting small duodenal and primary or metastatic nodal gastrinomas indeed found that primary duodenal tumors were missed more frequently than nodal metastasis at scintigraphy [2]. In the present case it is therefore much plausible that the gastric gastrinoma was of small size at the time of diagnosis of ZES and was not recognized at scintigraphy.

The occurrence of a gastrinoma in our patient was associated with the development of three other primary digestive tumors: a benign c-kit positive gastric stromal tumor and a duodenal villous adenoma concurrent of the diagnosis of the ZES and a gastric adenocarcinoma several years later. It has been reported that patients diagnosed with carcinoid tumors are at increased risk for the development of concurrent malignancies, especially gastrointestinal tumors $[1,4,5,11]$. In a series of 2837 carcinoid cases concurrent and nonconcurrent neoplasms were found, respectively, in $7 \%$ and $4 \%$ of the patients studied [6]. Carcinoid tumors in vitro can produce substances with a growth-promoting effect, and this might account for the increased incidence of tumors in patients with carcinoids. Gastrin has a trophic effect on the gastric mucosa, and its uncontrolled production might promote cell proliferation and neoplastic transformation in the adjacent mucosa by a direct paracrine effect. Indeed, this rare case brings additional support to this hypothesis as all associated neoplasms were found in the vicinity of the gastric neuroendocrine tumor. The generally low incidence of adenomas in the small intestine and stomach argues against unrelated events. Moreover, the absence of endoscopic evidence of gastric tumor at the time of diagnosis of the ZES indicates that the development of the villous tumor was subsequent to that of the gastrinoma.

In conclusion, this is the first report of a gastric collision tumor functionally expressing as ZES. The additional finding of associated neoplasms in the immediate vicinity of the gastrinoma emphasizes the possible implication of gastrin in tumor growth.

\section{Acknowledgements}

The authors thank L.M. Dupuis and O. Hougrand for their excellent technical assistance. L.d.L. is a Research Associate of the Belgian National Fund for Scientific Research.

\section{References}

1. Brown N, Smith M (1973) Neoplastic diathesis of patients with carcinoid. Cancer 32:216-222

2. Cadiot G, Lebtahi R, Sarda L, Bonnaud G, Marmuse J, Vissuzaine C, Ruszniewski P, Le Guludec D, Mignon M (1996) Preoperative detection of duodenal gastrinomas and peripancreatic lymph nodes by somatostatin receptor scintigraphy. Gastroenterology 111:845-854

3. Corsi A, Bosman C (1995) Adenocarcinoma and atypical carcinoid: morphological study of a gastric collision-type tumour in the carcinoma-carcinoid spectrum. Ital J Gastroenterol 27: 303-308

4. Declich P, Tavani E, Bellone S, Porcellati M, Raimondi D, Grassini R, Carneiro F, Bordi C (2002) Sporadic, syndromic and ZollingerEllison syndrome associated fundic gland polyps consistently express cytokeratin 7. Virchows Arch 441: 96-97

5. Gerstle J, Kauffman G, Koltun W (1995) The incidence, management, and outcome of patients with gastrointestinal carcinoids and second primary malignancies. J Am Coll Surg 180:427-432

6. Godwin II J (1975) Carcinoid tumors: an analysis of 2837 patients. Cancer 36:560-569

7. Klöppel G, Clemens A (1996) The biological relevance of gastric neuroendocrine tumors. Yale J Biol Med 69:69-74 
Published in : Virchows Archiv : An international Journal of Pathology (2002) Status : Postprint (Author's version)

8. Lewin K, Riddel R (1992) Endocrine cells. In: Lewin KJ (ed) Gastrointestinal pathology and its clinical implications. Igaku-Shoin, NewYork, pp 197-257

9. Liu T, Zhong S, Chen Y, Lin Y, Chen J, Li D, Wang DT, Gu CF, Yie SF (1989) Gastric gastrinoma. Chinese Med J 102: 774-782

10. Parks T (1970) Malignant carcinoid and adenocarcinoma of the stomach. Br J Surg 57:377-379

11. Rosenberg J, Welch J (1985) Carcinoid tumors of the colon: a study of 72 patients. Am J Surg 149:775-779

12. Royston C, Garnham J, Stagg B, Polak J (1972) The Zollinger-Ellison syndrome due to an infiltrating tumour of the stomach. Gut 13:638-642

13. Thompson N, Vinik A, Eckhauser F, Strodel W (1985) Extra-pancreatic gastrinomas. Surgery 98:1113-1120

14. Wolfe M, Jensen R (1987) Zollinger-Ellison syndrome. Current concepts in diagnosis and management. N Engl J Med 317:1200-1208

15. Yamashina M, Flinner R (1985) Concurrent occurrence of adenocarcinoma and carcinoid tumor of the stomach: a composite tumor or collision tumors? Am J Clin Pathol 83:233-236

16. Zollinger R, Ellison E (1955) Primary peptic ulcerations of the jejunum associated with islet cell tumors of the pancreas. Ann Surg $142: 708-729$ 